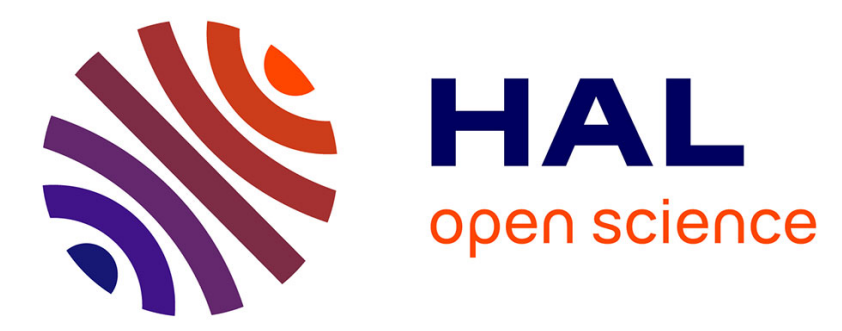

\title{
Dissipated energy and boundary condition effects associated to dry friction on the dynamics of vibrating structures
}

Baraa Al Sayed, Eric Chatelet, Sébastien Baguet, Georges Jacquet-Richardet

\section{- To cite this version:}

Baraa Al Sayed, Eric Chatelet, Sébastien Baguet, Georges Jacquet-Richardet. Dissipated energy and boundary condition effects associated to dry friction on the dynamics of vibrating structures. Mechanism and Machine Theory, 2011, 46, pp.479-491. 10.1016/j.mechmachtheory.2010.11.014 . hal01569162

\author{
HAL Id: hal-01569162 \\ https://hal.science/hal-01569162
}

Submitted on 26 Jul 2017

HAL is a multi-disciplinary open access archive for the deposit and dissemination of scientific research documents, whether they are published or not. The documents may come from teaching and research institutions in France or abroad, or from public or private research centers.
L'archive ouverte pluridisciplinaire HAL, est destinée au dépôt et à la diffusion de documents scientifiques de niveau recherche, publiés ou non, émanant des établissements d'enseignement et de recherche français ou étrangers, des laboratoires publics ou privés.

\section{(c)(1)}

Distributed under a Creative Commons Attribution| 4.0 International License 


\title{
Dissipated energy and boundary condition effects associated to dry friction on the dynamics of vibrating structures
}

\author{
Baraa Al Sayed, Eric Chatelet, Sébastien Baguet, Georges Jacquet-Richardet \\ Université de Lyon, CNRS, INSA-Lyon, LaMCoS UMR5259, France
}

In turbomachines, dry friction devices (under platform dampers, shrouds, and tie-wire) are usually introduced to reduce resonant responses of bladed disks. Dry friction between rubbing elements induces a highly nonlinear dynamic behaviour which flattens the frequency response functions. It is clear that such behaviour requires an optimisation process to find the optimum parameters that lead to the minimum forced response amplitudes. However, different interpretations still remain concerning the explanation of the physical origin of this type of flattening. The most common one is based on dissipated energy. In this case, heat resulting from the relative frictional motion between contacting surfaces is supposed to bring sufficient dissipation to flatten response functions. On the other hand, a different approach considers that a decrease in vibrational amplitudes is explained by changes in boundary conditions induced by a stick/slip behaviour. In this study, a single degree-of-freedom system is used and analysed both in time and in frequency domains (Harmonic Balance Method) in order to show the contribution of respectively energy dissipation and change of contact state on peak levels.

\author{
Keywords: \\ Dry friction \\ Peak flattening \\ Multi Harmonic Balance Method \\ Dissipated energy \\ Boundary conditions
}

\section{Introduction}

Devices involving dry friction [1,2] are often used to decrease maximal amplitudes of structures during resonance. Each device is characterised by a different behaviour depending on the geometry, as well as the position and the dimensions of surfaces in contact. As a consequence, different adapted contact models have been developed, as presented for example in [3-6]. Two main categories of friction contact models are encountered: Macroslip and Microslip [7]. The main difference between them concerns the homogeneity of their behaviour along contact surfaces. With a Macroslip model, the behaviour of the contact surface is global and homogeneous and every contact at any moment is either in a completely slipping or in a completely stuck state. Considering Microslip models, the behaviour of contact surfaces is no more homogeneous and mixed configurations may be present. Part of the contact surface may be stuck while another part may slip at the same time. Taking into account the most adapted model, a good agreement can be obtained between numerical and experimental results, as shown for example in [8-10].

A common characteristic between all friction devices relies on the induced flattening of frequency response function (FRF) peaks obtained when specific normal loads are applied at the contact. When normal load is very high in comparison with the excitation force, the dynamic behaviour is almost linear and FRF exhibits high level resonance peaks. For lower normal loads, the amplitude of peaks diminishes and the FRF may become very flat. Finally, when normal load is much lower than the excitation force, the dynamic behaviour is linear again and the FRF may exhibit high amplitude peaks [11,12].

Two different approaches are used to explain such flattening. Energetic approaches, as presented for example in [13], suppose a direct relationship between peak flattening and the energy dissipated by dry friction. Experimentally it is shown that, within stick/ slip states, the amplitudes of FRF decrease and the ratio between dissipated energy and input energy increases. Consequently 
dissipated energy is considered as the cause of flattening. On the other hand, peak flattening may be explained as a consequence of changes in boundary conditions during stick/slip states. The occurrence of different contact states induces a phase difference between displacement and components of the nonlinear friction force that limit its effect and then explain peak flattening. Several authors [14-16] analyse bending and/or torsion stick/slip motions associated to industrial applications.

In order to get a better understanding of this phenomenon, a numerical model based on a single dof system is used here. An energetic analysis and an analysis based on the equilibrium of forces at resonance are both considered. A classical Multi Harmonic Balance Method (MHBM) operating in the frequency domain [17-19] compared to a reference transient solution obtained in the time domain is used to determine the influence of dissipated energy and changes in boundary conditions associated to contact states.

\section{Developments and contact model}

The prediction of responses of systems involving dry friction is complex due to their highly nonlinear behaviour. Different techniques are available to solve such nonlinear problems. Time domain solutions, based on numerical integrations, give precise results but are often costly and may induce large discrepancies if time steps are not properly chosen. Frequency based methods generally reduce drastically computational efforts by considering that the solution is periodic. Different approaches may be used, depending on the complexity of the structure and the level of non linearity. The classical Harmonic Balance Method is used in many applications dealing with the dynamics of periodically excited structures with contact and dry friction. Steady state response approximations of motion are obtained in the frequency domain by decomposing the periodical response of the nonlinear system into a Fourier series, leading to a set of algebraic equations. The accuracy of this method to predict responses and friction forces is highly dependent upon the number of harmonics retained. But keeping a too large number of harmonics reduces the interest of the method.

\subsection{Multi Harmonic Balance Method}

A single dof system (Fig. 1) associated with a Masing Macroslip contact model (Fig. 2) [18,19]) has been chosen in order to simplify the numerical procedures and to be able to conduct parametric studies. This system is supposed to model a blade to ground with Macroslip frictional contact where parts in contact are either slipping or sticking. Contact separation and partial slips are not allowed here.

A periodic excitation force $F_{e x}(t)=f_{0} \cos \omega \mathrm{t}$ is applied to the system, and a normal load $F_{n}$ is applied to the frictional damper to load the contact.

For such systems, the motion equation is:

$$
m \ddot{x}+c \dot{x}+k x=F_{e x}-f_{n l} .
$$

Structural parameters are: mass $m$, stiffness $k$ and viscous damping $c$. The frictional device is represented by its stiffness $k_{d}$, the coefficient of friction $\mu$ at the contact interface and the normal load $F_{n}$ which is supposed to be constant. $x(t)$ represents the time dependent displacement of the entire system while $z(t)$ is the displacement of the contact point with respect to ground. $y(t)$ is the relative displacement between the mass and the contact point $y(t)=x(t)-z(t)$.

According to the Macroslip approach [19], the friction force $f_{n l}$ can be expressed as:

$$
f_{n l}(t)=\left\{\begin{array}{l}
+k_{d}(x(t)-z(t)) \text { when } k_{d}|x-z| \leq \mu F_{n} \\
+\mu F_{n} \operatorname{sign}(\dot{z}) \text { when } k_{d}|x-z| \geq \mu F_{n}
\end{array}\right.
$$

The steady state associated to Eq. (1) may be obtained using the MHBM, which considers the total displacement as a superposition of harmonic components with frequency $\omega$.

$$
x(t)=\sum_{n=1}^{N} a_{n} \cos (n \theta)+b_{n} \sin (n \theta)
$$

where $\theta=\omega t, a_{n}$ and $b_{n}$ are harmonic (cosine and sine) components and $N$ is the number of harmonic retained.

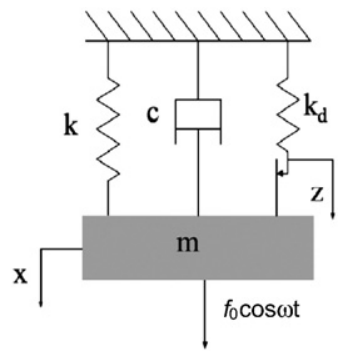

Fig. 1. 1 DOF system. 


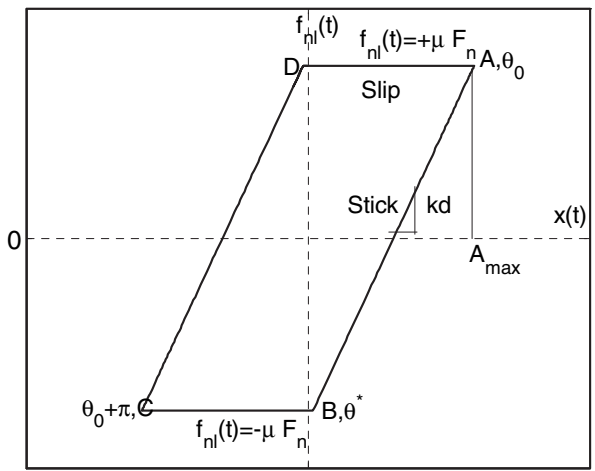

Fig. 2. Hysteresis loop (Masing Model): Multi term approximation. Parameters $\theta^{*}$ and $\theta_{0}$.

Wang [19] shows that the friction force, when associated with the Macroslip model of Fig. 2, can be approximated as follows:

$$
f_{n l}(t)=\left\{\begin{array}{l}
\mu F_{n}+k_{d}\left(x(t)-A_{\max }\right) \text { for stick contact state } \\
\mu F_{n} \operatorname{sign}(\dot{x}) \text { for slip contact state }
\end{array}\right.
$$

where $A_{\max }$ is the highest amplitude of the total displacement and $x(t)$ is given as a function of $\theta_{0}$.

$$
A_{m}=A_{\max }=x\left(\theta_{0}\right)=\sum_{n=1}^{N} a_{n} \cos \left(n \theta_{0}\right)+b_{n} \sin \left(n \theta_{0}\right)
$$

The two parameters $\theta^{*}$ and $\theta_{0}$ are introduced to determine whether the slider is within a stick or a slip state. Angle $\theta_{0}$ is associated to point $\mathrm{A}$ of the hysteresis cycle where the slip state stops and the stick state begins. Angle $\theta^{*}$ is associated to point $\mathrm{B}$ where the slip state begins and the stick state stops. Taking into account these two parameters, defining contact states, the nonlinear force can be developed into two different expressions, one for the stuck state where $\theta_{0}<\theta<\theta^{*}$ and another for the slip state where $\theta^{*}<\theta<\theta_{0}+\pi$.

As observed in Figs. 2 and 3, the stuck state is characterised by line (AB) and the slipping state by line (BC).

Changes in contact states from slip to stick are identified by points $A$ and $C$, at $\theta=\theta_{0}$ and $\theta=\pi+\theta_{0}$ respectively. Change from stick to slip occurs at point B where $\theta=\theta^{*}$.

The nonlinear force due to dry friction is also approximated using a truncated Fourier series as:

$$
f_{n l}(t)=\sum_{n=1}^{N} f c_{n}(\theta) \cos (n \theta)+f s_{n}(\theta) \sin (n \theta)
$$

$f c_{n}(\theta)$ and $f s_{n}(\theta)$ are functions of the contact model chosen and, as the nonlinear force is antisymmetric [19], can be obtained from the following Fourier integrals:

$$
f c_{n}(\theta)=\frac{2}{\pi} \int_{\theta_{0}}^{\theta_{0}} f_{n l}^{+\pi}(t) \cos (n \theta) d \theta
$$

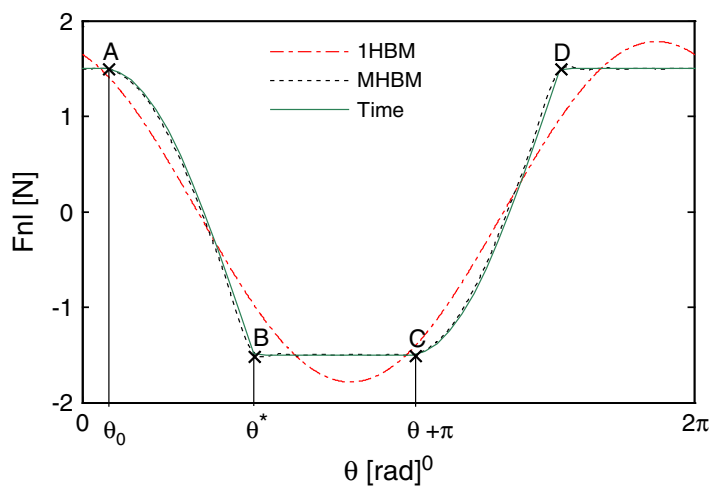

Fig. 3. Nonlinear force versus time in 1HBM, MHBM and time solution for $F_{n}=3 \mathrm{~N}$, frequency $=20 \mathrm{~Hz}$. 


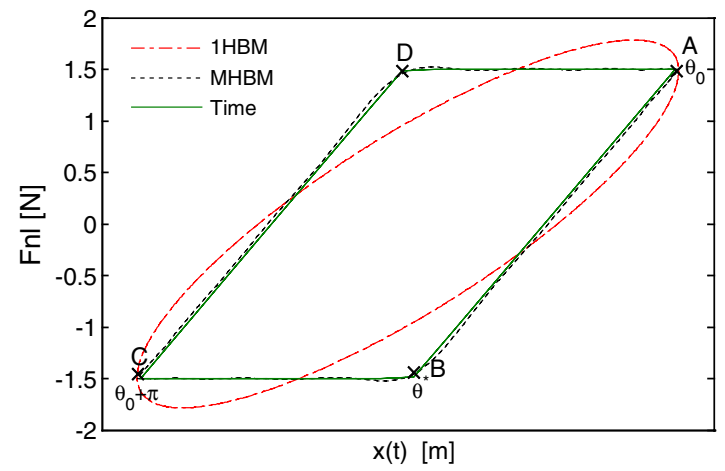

Fig. 4. Hysteresis loop given by $1 \mathrm{HBM}, \operatorname{MHBM}(\mathrm{N}=55)$ and time solution for $F_{n}=3 \mathrm{~N}$, frequency $=20 \mathrm{~Hz}$.

$$
f s_{n}(\theta)=\frac{2}{\pi} \int_{\theta_{0}}^{\theta_{0}} f_{n l}(t) \sin (n \theta) d \theta
$$

Both expressions (2) of the nonlinear force in stuck and slip states, written as a function of the state parameters $\theta_{0}$ and $\theta^{*}$, are considered when integrating over the proper intervals.

By introducing ( 3 ) and its derivatives and (4) into the equation of motion (1) and identifying the cosine and sine components, a system of $\mathrm{N}$ algebraic equations with $2 \mathrm{~N}+2$ unknowns $a_{1}, b_{1}, \ldots ., a_{n}, b_{n}, \theta_{0}, \theta^{*}$ is obtained.

$$
\begin{aligned}
& -m \omega^{2}\left[\sum_{n=1}^{N} n^{2}\left(a_{n} \cos (n \theta)+b_{n} \sin (n \theta)\right)\right] \\
& -c\left[\sum_{n=1}^{N} n\left(a_{n} \sin (n \theta)-b_{n} \cos (n \theta)\right)\right] \\
& +k\left[\sum_{n=1}^{N} a_{n} \cos (n \theta)+b_{n} \sin (n \theta)\right]+\sum_{n=1}^{N} f c_{n} \cos (n \theta)+f s_{n} \sin (n \theta)=f_{0} \cos (\theta)
\end{aligned}
$$

Two additional equations are required. The first one states that at $\theta=\theta_{0}$, displacement $x(t)$ reaches a maximal value $A_{\max }$ and so, velocity is null. Null velocity is written as:

$$
\left.\frac{\partial x}{\partial t}\right|_{\theta=\theta_{0}}=\omega \sum_{n=1}^{N} n\left(b_{n} \cos \left(n \theta_{0}\right)-a_{n} \sin \left(n \theta_{0}\right)\right)=0 .
$$

At point B (Fig. 2), the two expressions of the nonlinear force given for stick and slip states are equal. Consequently, the second additional equation will be:

$$
\begin{aligned}
& \mu F_{n}+k_{d}\left(x(t)-A_{m}\right)=-\mu F_{n} \text { then } \\
& \mu F_{n}+k_{d} \sum_{n=1}^{N}\left(a_{n}\left(\cos \left(n \theta^{*}\right)-\cos \left(n \theta_{0}\right)\right)+b_{n}\left(\sin \left(n \theta^{*}\right)-\sin \left(n \theta_{0}\right)\right)\right) \\
& =-\mu F_{n} .
\end{aligned}
$$

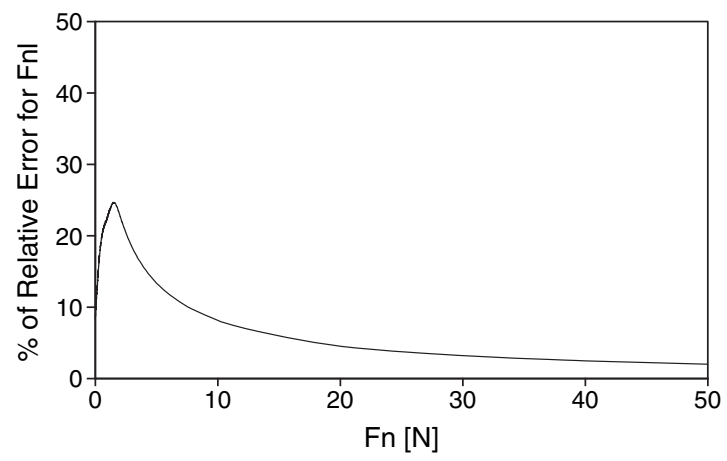

Fig. 5. Relative error for the nonlinear force calculated with 1HBM and MHBM. 
For each pulsation $\omega$, initial values are needed for $\theta^{*}$ and $\theta_{0}$. The unknown coefficients $a_{n}, b_{n}, \theta^{*}$ and $\theta_{0}$ are determined by solving Eqs. (9)-(11) using a Newton-Raphson iterative method [19].

\subsection{MHBM versus time solution}

Parameters considered for the numerical application are as follows: mass $m=1.24 \mathrm{~kg}$, system stiffness $k=17,890 \mathrm{~N} / \mathrm{m}$ and viscous damping $c=0.134 \mathrm{Ns} / \mathrm{m}$. Contact stiffness $k_{d}=3500 \mathrm{~N} / \mathrm{m}$, and dry friction coefficient is $\mu=0.5 . f_{0}$ is taken to unity.
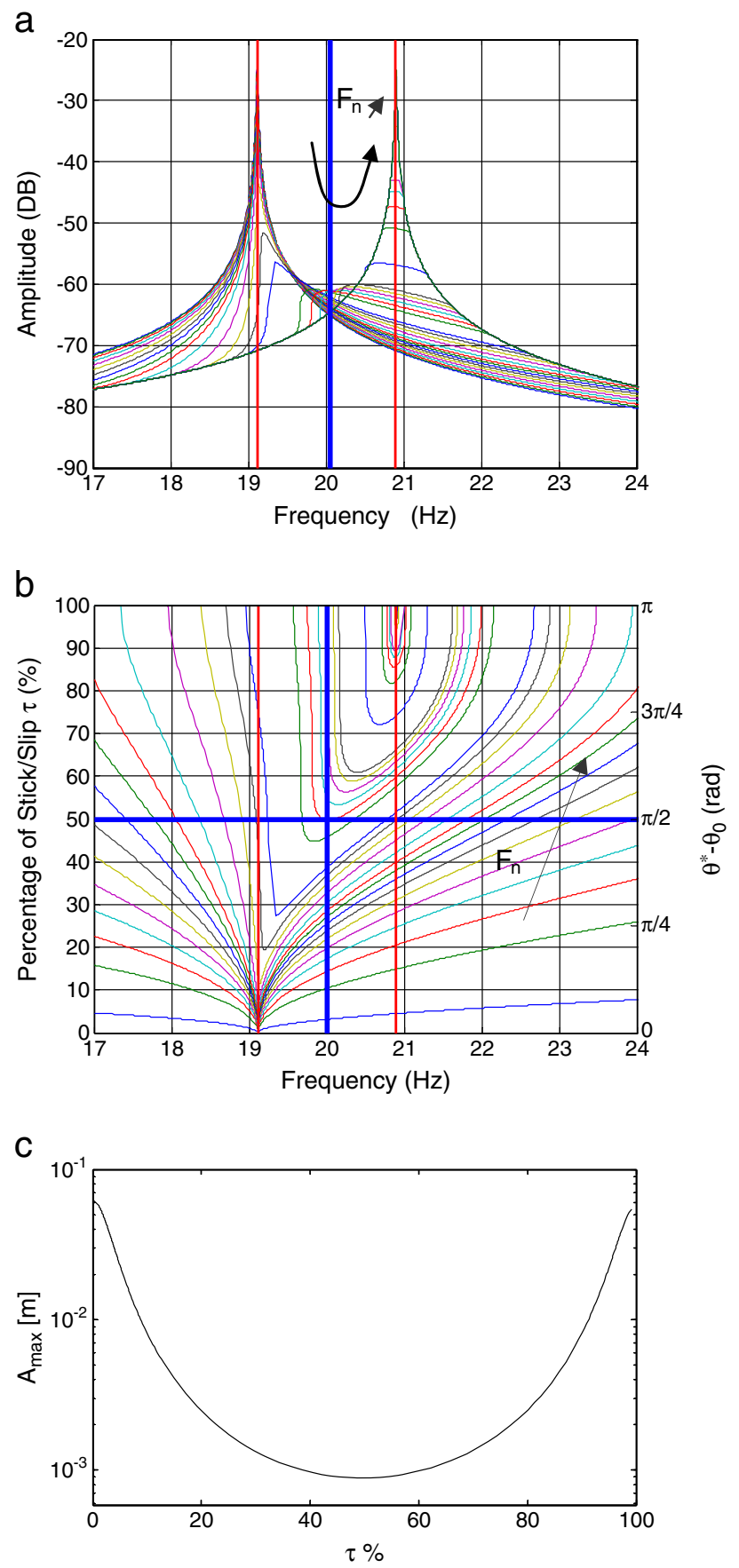

Fig. 6. a) Frequency response functions for different normal contact loads obtained using the MHBM, b) percentage of stick/slip over a period, and c) evolution of maximum displacement $A_{\max }$ with respect to parameter $\tau$. 


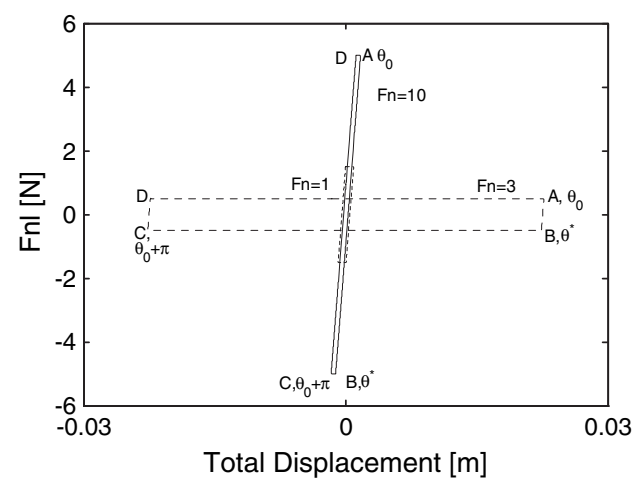

Fig. 7. Shape of hysteresis loop for the three contact states.

Figs. 3 and 4 show the evolution of the nonlinear force as a function of time and displacement respectively. Results obtained from the MHBM (using $\mathrm{N}=1$ and $\mathrm{N}=55$ harmonics) are compared with those obtained from a time marching direct solution (Newmark method with constant average acceleration). The Multi Harmonic Balance truncated at the first harmonic will be denoted as 1HBM and MHBM will be associated to cases when more than one harmonic are retained.

As expected, the accuracy of the MHBM increases with the number of harmonics considered.

Fig. 5 shows the error obtained for the nonlinear force $f_{n l}(t)$ when using the 1HBM as a function of the normal load (reference results obtained from MHBM with $\mathrm{N}=55$ ).

This error is $10 \%$ at the quasi slip state, increases up to $25 \%$ and finally decreases to zero when approaching the quasi stick region corresponding to the high values of normal force $F_{n}$. Then, the 1HBM provides an accurate description of hysteresis curves as well as nonlinear forces, especially for low values of the normal contact force leading to a highly nonlinear behaviour.

\subsection{Physical considerations}

Fig. 6a gives the FRFs obtained at different normal loads using the MHBM.

For $F_{n}=0.01 \mathrm{~N}$, contact is always sliding and the system exhibits a linear behaviour. In this case, the equivalent stiffness is $k_{e q}=k$ and resonance occurs at:

$$
f_{1}=\frac{1}{2 \pi} \sqrt{\frac{k e q}{m}}=19.1 \mathrm{~Hz}
$$

For $F_{n}>500 \mathrm{~N}$, contact is always completely stuck and the resonant frequency is in this case a function of both system stiffness and contact stiffness. The equivalent stiffness is $k_{e q}=k+k_{d}$ and resonance is at:

$$
f_{2}=\frac{1}{2 \pi} \sqrt{\frac{k_{e q}}{m}}=\frac{1}{2 \pi} \sqrt{\frac{k_{d}+k}{m}}=20.9 \mathrm{~Hz}
$$

When normal load decreases from $500 \mathrm{~N}$ to $0.01 \mathrm{~N}$, contact state changes from stick to stick/slip state. As a consequence of those changes, peaks become flat and the amplitudes obtained are much smaller than those associated to the linear cases when

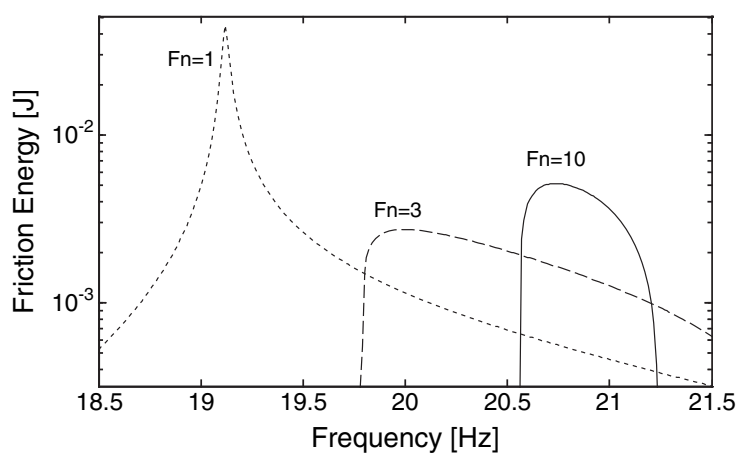

Fig. 8. Dry friction energy for different normal loads. 


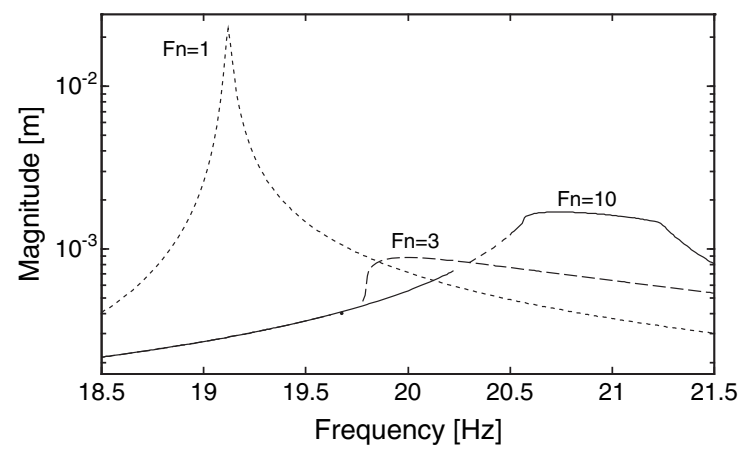

Fig. 9. Response amplitudes for different normal loads.

contact is stuck or slipping. The dynamic behaviour obtained with a simple single dof system with dry friction exhibits the three different contacts states (stick, stick/slip and slip) and peak flattening is classically observed.

The advantage of representing results with respect to the difference between parameters $\theta^{*}$ and $\theta_{0}$ lies in an easier comprehension of shape changes induced on the hysteresis curves during various contact states.

An illustration of the transformation of hysteresis curves is given in Fig. 7. The case of slip contact induces a small difference between $\theta^{*}$ and $\theta_{0}$ and, graphically, when approaching this state, point $\mathrm{B}$ becomes closer to point $\mathrm{A}$ than to point $\mathrm{C}$ (Fig. 2). As a consequence, the hysteresis curve degenerates into a horizontal line. When approaching the stick state, difference in the two parameters comes close to $\pi$, and point B becomes closer to point C. In this case, the hysteresis curve evolves toward a line with a $k_{d}$ slope. The percentage of the stick state $\tau$ over a period of motion is defined as $\tau=100 .\left(\theta^{*}-\theta_{0}\right) / \pi$. It is equal to zero for systems without friction and increases with the increase in contact normal force. $\tau=0 \%$ and $\tau=100 \%$ correspond to pure slip and pure stick motions respectively.

The maximal efficiency of the considered damping device, corresponding to the minimum amplitude in Fig. $6 \mathrm{c}$ is observed at a frequency of $20 \mathrm{~Hz}$ and a normal force $F_{n}=3 \mathrm{~N}$. According to Fig. $6 \mathrm{~b},\left(\theta^{*}-\theta_{0}\right)$ is in this case equal to $\pi / 2$, then $\tau=50 \%$. This specific
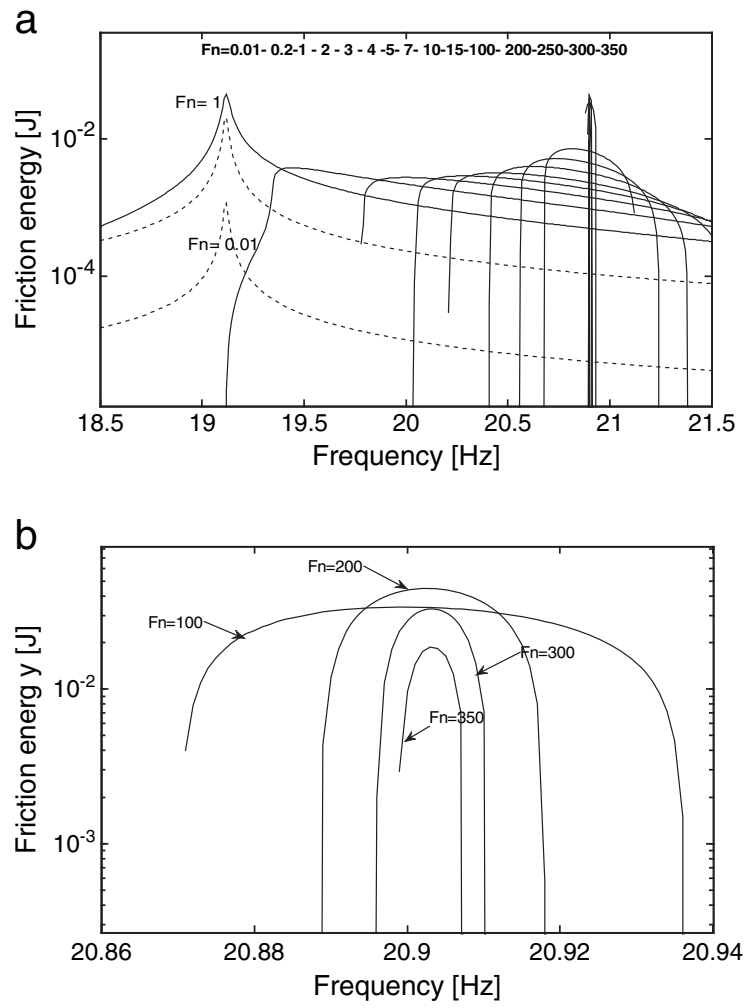

Fig. 10. a) Dissipated energy by dry friction for different normal loads. b) Dissipated energy by dry friction for $F_{n}=100-200-300-350$ N. 


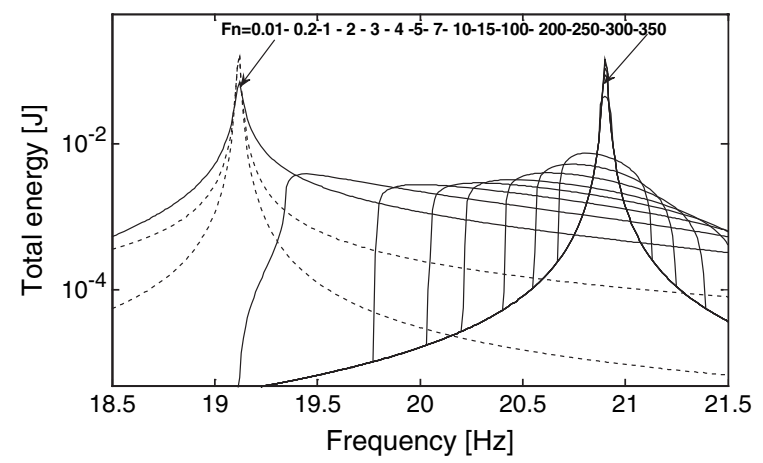

Fig. 11. Total dissipated energy for different normal loads.

state corresponds to a semi stick semi slip configuration. Fig. 6c combines the results of Fig. 6a and b. Fig. 6a shows that for a given normal force $F_{n}$, there exist only one maximum amplitude $A_{\max }$ and an associated frequency. From Fig. 6b, it appears that at this frequency, are associated a unique set of contact parameters $\theta^{*}$ and $\theta_{0}$, and consequently a unique value of $\tau$. Fig. $6 c$ gives the evolution of the maximal amplitude calculated for each normal load as a function of parameter $\tau$. Maximal efficiency is clearly obtained for $\tau=50 \%$.

\section{Dry friction and viscous dissipated energy}

The energy dissipated by the dry friction damper $E_{\text {friction }}$ and by the viscous damper $E_{\text {viscous }}$ is calculated over a period of one forcing cycle at steady state, using the following expressions:

$$
\begin{aligned}
& E_{\text {friction }}=\int_{T} f_{n l}(t) \dot{y} d t \\
& E_{\text {viscous }}=\int_{T} F_{\text {viscous }} \dot{x} d t=\int_{T} c(\dot{x})^{2} d t=\pi c \omega\left(A_{\max }(\omega)\right)^{2}
\end{aligned}
$$

where $f_{n l}(t)$ is the nonlinear contact force, $y(t)$ the relative displacement between damper and mass, and $A_{\text {max }}$ the amplitude of the total displacement $x(t)$ associated with forcing frequency $\omega$.

The dissipated energy by dry friction may be obtained from the hysteresis loop giving the nonlinear friction force $f_{n l}(t)$ as a function of the relative displacement $y(t)$. Relations between response functions and dry friction dissipated energy are illustrated in Figs. 8-10. Those results are associated to three given normal forces $F_{n}$ that induce the three specific contact states: majority slip for $F_{n}=1 \mathrm{~N}$, stick/slip for $F_{n}=3 \mathrm{~N}$ and finally $F_{n}=10 \mathrm{~N}$ for majority stuck. The case $F_{n}=1 \mathrm{~N}$ gives the highest magnitude of response, the largest hysteresis curve and the highest dry energy dissipation. $F_{n}=3 \mathrm{~N}$ gives the lowest amplitude associated to the thinnest hysteresis curve, whereas $F_{n}=10 \mathrm{~N}$ leads to greater levels in magnitude.

Fig. 10a shows the energy dissipated by dry friction for different normal loads varying from $0.01 \mathrm{~N}$ to $350 \mathrm{~N}$. At very low values of normal loads, the system is purely slipping. As the system is purely slipping, dry friction energy dissipation tends to zero. For very high normal loads $F_{n}>350 \mathrm{~N}$, relative motion is no more possible (stuck state) and dry energy dissipation tends to zero as well. There is no dissipated energy by dry friction associated to those two limit linear cases.

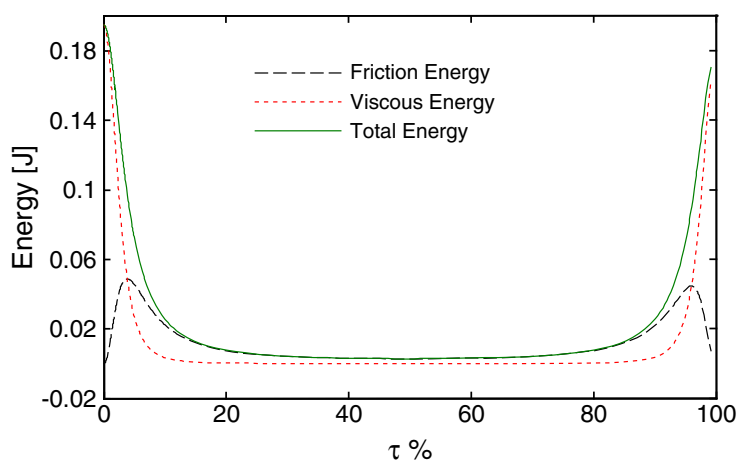

Fig. 12. Evolution of total dissipated energy as a function of parameter $\tau$ associated to the maximal displacement. 


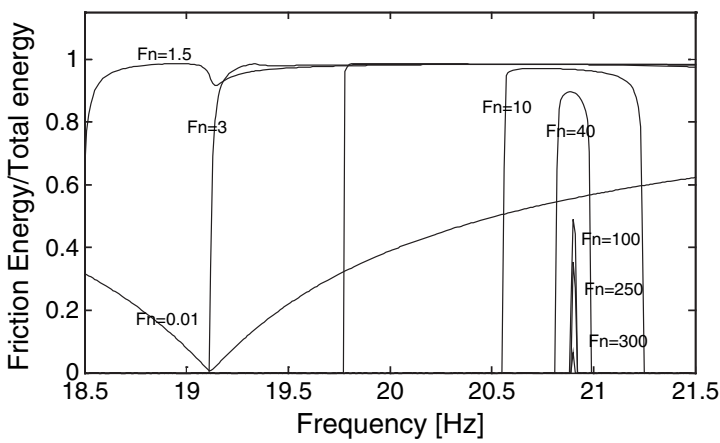

Fig. 13. Ratio of dissipated energy by dry friction $E_{\text {friction }}$ to total dissipated energy $E_{\text {tot }}$ as a function of frequency for different normal loads.

The total dissipated energy is obtained by adding dissipation due to dry friction $E_{\text {friction }}$ and viscous damping $E_{\text {viscous }}$ as expressed in (16).

$$
E_{\text {tot }}=E_{\text {friction }}+E_{\text {viscous }}
$$

Fig. 11 gives the evolution of the total dissipated energy and the same conclusions as made previously appear clearly. Dissipated energy decreases also with peak amplitude lowering (Fig. 6a).

Fig. 12 superimposes the evolution the maximum of the total energy, the dry friction energy and the viscous energy (obtained from Figs. 10a and 12) as a function of $\tau$. Using this representation, low normal loads are located near $\tau=0 \%$ whereas high normal loads are located near $\tau=100 \%$.

It is interesting to examine the ratio of energies associated to dry friction and to viscous damping (Fig. 13). When normal load is low, contact is mainly slipping and the rubbing element becomes prominent and can dissipate more than 95\% of the total dissipated energy. Within stick/slip states the influence of the rubbing element is much more significant than the influence of the viscous damper. Viscous damping energy is almost negligible within stick/slip zone and participates significantly only for the quasi linear cases associated to low and high normal loads.

Energy dissipated by dry friction exhibits two maxima, corresponding to $F_{n}=1$ and $F_{n}=200 \mathrm{~N}$ (Fig. 10). These maxima are also associated to the resonance peaks, as observed in Fig. 6a. Below $F_{n}=1 \mathrm{~N}$, the system tends to a pure slip behaviour and so $E_{\text {friction }}$ tends to zero (Fig. 10a). Beyond $F_{n}=200 \mathrm{~N}$, the system tends to pure stick behaviour and $E_{\text {friction }}$ tends also to zero (Fig. 10b). $E_{\text {friction }}$ has a minimum value at the optimum value of the stick-slip states, corresponding to the minimum displacement amplitude, observed for $\tau=50 \%$ in Fig. $6 c$.

The total energy is also minimum for $\tau=50 \%$, and globally follows the same evolution than the maximal amplitude response of Fig. 6c.

As a conclusion, since the minimum in displacement does not correspond to the maximum of dissipated energy, neither total dissipated energy nor energy only dissipated by dry friction is sufficient to explain FRF flattening.

\section{Changes in the contact state due to stick/slip behaviour}

The MHBM has a major interest here as it allows defining a phase parameter that describes changes in boundary conditions within stick/slip motions. The nonlinear tangential force can be conveniently expressed as:

$$
f_{n l}(t)=\sum_{n=1}^{N} f c_{n} \cos (n \theta)+\sum_{n=1}^{N} f s_{n} \sin (n \theta)=f_{n l c o s}(n \theta)+f_{n l \sin }(n \theta) .
$$

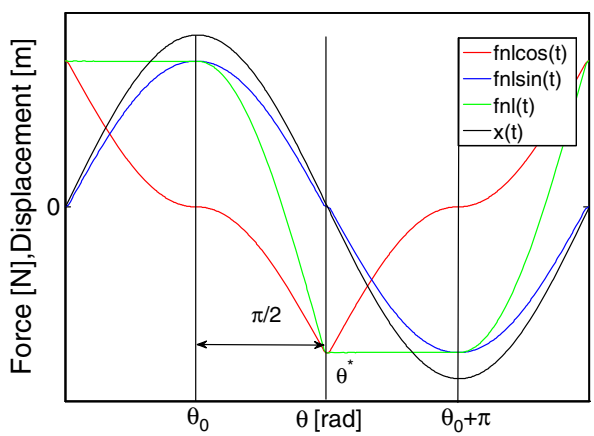

Fig. 14. $\mathrm{f}_{\text {nlcos }}(t), f_{\text {nlsin }}(t), f_{n l t}(t)$, and $x(t)$ as function of angle $\theta$. 


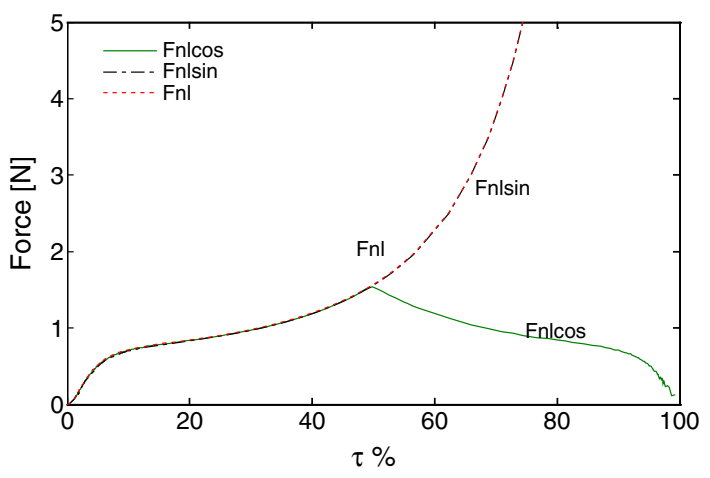

Fig. 15. In-phase and out-of-phase components of the nonlinear force with the MHBM.

Fig. 14 shows the same evolution for $f_{n l}(t)$ over a cycle period $2 \pi$ (as drawn in Fig. 3 ), its cosine and sine components $f_{\text {nlcos }}(t)$, $f_{n l s i n}(t)$ and displacement $x(t)$. This figure demonstrates that displacement $x(t)$ and nonlinear force $f_{n l}(t)$ are in phase, with maxima at $\theta=\theta_{0}$, as defined for the Macroslip model presented in Fig. 2. The sine component is also in phase with $x(t)$. On the other hand, the cosine part of the nonlinear force is confirmed as out-of-phase with respect to displacement. These results stand whatever the value of the normal force $F_{n}$ and the value of frequency, i.e. whatever the value of $\tau$.

Fig. 15 gives the evolution of the nonlinear tangential force $f_{n l}$ as well as the in-phase $F_{n l s i n}$ and out-of-phase $F_{n l c o s}$ components of the nonlinear force calculated using the MHBM $(\mathrm{N}=55)$, as a function of parameter $\tau$. For low normal forces, and then for low values of stick percentage $\tau$ close to $0 \%$, the magnitude of the nonlinear force starts at zero (totally slip state). Both $F_{n l c o s}$ and $F_{n l s i n}$ have the same contribution until reaching the optimum of the stick-slip states at $\tau=50 \%$.

At this point, $F_{\text {nlcos }}$ begins to decrease and the nonlinear force increases and becomes almost equal to $F_{n l s i n}$ when approaching the stick state. Close to the stick state $(\tau=100 \%), f_{n l}(t)$ is almost equal to the magnitude of its in-phase component $F_{n l s i n}$ whereas the magnitude of the out-of-phase component $F_{\text {nlcos }}$ is almost equal to zero.

This phenomenon can be physically explained as follows: close to the stick state, the nonlinear force becomes linear and in phase with displacement; implying that the contribution of the in-phase component will be dominant whereas the contribution of the out-of-phase component will be negligible.

When examining all the forces acting, the viscous force and the cosine component of the nonlinear force are always out-ofphase with respect to displacement, whereas the inertial force, the spring force and the sine component of the nonlinear force are in phase. Peak flattening is measured at the maximal amplitude $A_{\max }$ that occurs at a particular value of frequency $\omega$ denoted $\Omega$. The in-phase forces with respect to displacement $x(t)$ are:

$$
\begin{aligned}
& \text { Inertial Force }: f_{i n}(t)=m \ddot{x}(t)=-m \Omega^{2} x(t) \\
& \text { Restitution Force }: f_{\text {spring }}(t)=k \cdot x(t) \\
& \text { In-phase tangential Force }: f_{n l s i n}(t)=\sum_{n=1}^{N} f s_{n} \sin (n \theta) \text {. }
\end{aligned}
$$

Consequently, the maximal value of the total in-phase force $F_{p}$ associated to maximal amplitudes is:

$$
F_{p}=F_{\text {in }}+F_{\text {spring }}+F_{n l s i n}
$$

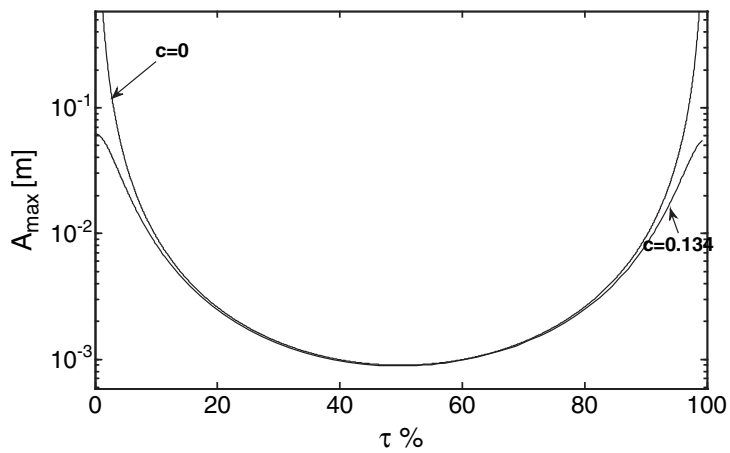

Fig. 16. Evolution of maximum displacement $A_{\max }$ with respect to $\tau$ for $c=0$ and $c=0.134$. 


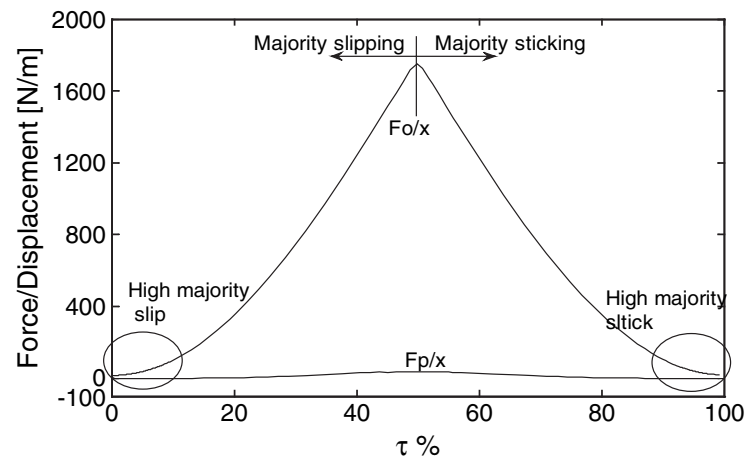

Fig. 17. Evolution of the in- and out-of-phase forces per displacement ( $F_{p / x}$ and $\left.F_{o / x}\right)$ with respect to $\tau$.

$$
\begin{aligned}
& F_{\text {in }}=-\|m \ddot{x}(t)\|, F_{\text {spring }}=k \cdot\|x(t)\|=k \cdot A_{\max }, \\
& F_{n l s i n}=\left\|\sum_{n=1}^{N} f s_{n} \sin (n \theta)\right\|=\left\|f_{n l s i n}(t)\right\|
\end{aligned}
$$

The out-of-phase forces are:

Viscous force : $f_{\text {visc }}(t)=c \dot{x}(t)$

Out-of-phase tangential Force :

$$
f_{\text {nlcos }}(t)=\sum_{n=1}^{N} f c_{n} \cos (n \theta)
$$

with a maximum $F_{o}$ at:

$$
\begin{aligned}
& F_{o}=F_{\text {visc }}+F_{n l c o s} \\
& F_{\text {visc }}=\|\dot{c}(t)\| F_{n l c o s}=\left\|\sum_{n=1}^{N} f c_{n} \cos (n \theta)\right\|=\left\|f_{\text {nlcos }}(t)\right\|
\end{aligned}
$$

These forces may be written per displacement unit $F_{p / x}$ and $F_{o / x}$ :

$$
\begin{aligned}
& F_{p / x}=k-\frac{\|m \dot{x}(t)\|}{A_{\max }}+\frac{F_{n l s i n}}{A_{\max }} \\
& F_{o / x}=\frac{\|c \ddot{x}(t)\|}{A_{\max }}+\frac{F_{n l c o s}}{A_{\max }}
\end{aligned}
$$

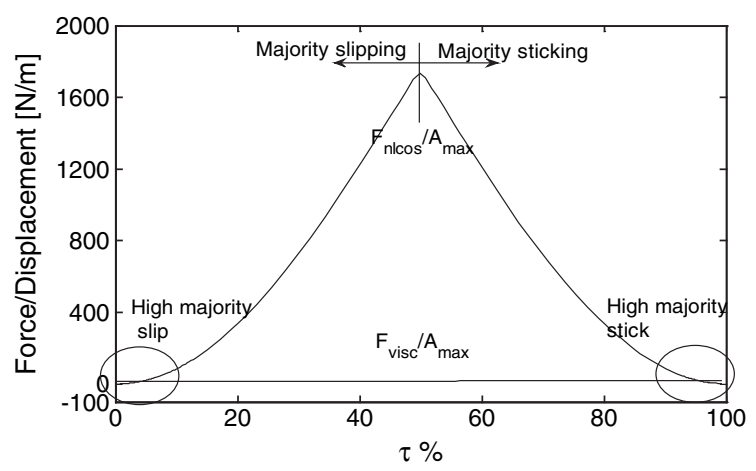

Fig. 18. Evolution of $\left(F_{\text {nlcos }} / \mathrm{A}_{\max }\right)$ and $\left(F_{\text {visc }} / \mathrm{A}_{\max }\right)$ components of the out-of-phase per displacement force, with respect to $\tau$. 


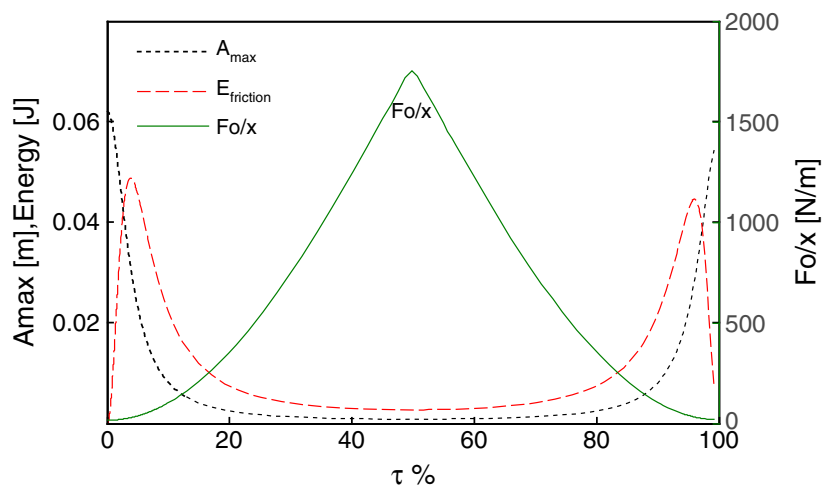

Fig. 19. Maximal amplitude, dry friction dissipated energy and out-of-phase force per displacement with respect to $\tau$.

Considering equilibrium, it becomes:

$$
\begin{aligned}
F_{\text {tot } / x} & =\sqrt{F_{o / x}^{2}+F_{p / x}^{2}} \\
& =\sqrt{\left(\frac{\|c \dot{x}(t)\|}{A_{\max }}+\frac{F_{n l c o s}}{A_{\max }}\right)^{2}+\left(k-\frac{\|m \ddot{x}(t)\|}{A_{\max }}+\frac{F_{\text {nlsin }}}{A_{\max }}\right)^{2}} \\
& =\frac{f_{0}}{A_{\max }}
\end{aligned}
$$

where $f_{0}$ is the excitation force. Fig. 16 presents the evolution of the amplitude $A_{\max }$ as a function of the stick percentage $\tau$. The highest amplitudes are obtained in the case of quasi linear slipping and stuck contact. The optimal state, associated to the lowest amplitude, is obtained at $\tau=50 \%$ where a period of oscillation is constituted by half-stuck and half-slip motions.

Fig. 17 shows the evolution of the in-phase and out-of-phase forces per displacement unit $F_{p / x}$ and $F_{o / x}$. The total in-phase force appears clearly negligible when compared to the out-of-phase component, excepted close to purely slip and stick states where both components have the same order of magnitude. The maximum value for $F_{o / x}$ occurs at $\tau=50 \%$ where the lowest maximal amplitude $A_{\max }$ has been noticed before.

Fig. 18 gives the two components of the total out-of-phase force $F_{o / x}$ associated to dry friction $F_{n l c o s} / A_{\max }$ and to viscous damping $F_{\text {visc }} / A_{\max }$. Viscous damping effects cannot be neglected close to completely stuck and to completely slipping contact states. Elsewhere, the total out-of-phase force $F_{o / x}$ is very well approximated by $F_{n l c o s} / A_{\max }$. This result is confirmed by Fig. 16 which shows the highest amplitude $A_{\max }$ as a function of the different contact states for both cases with and without viscous damping. Whatever the case, the optimal stick/slip states remains at $\tau=50 \%$ with about the same value of amplitude and viscous damping effect is only significant for the completely stuck or slipping states.

Fig. 19 superimposes the evolution of the maximal displacement amplitude, the dry friction dissipated energy $E_{\text {friction }}$ and the out-of-phase force per displacement unit $F_{o / x}$, given as function of the percentage of stick $\tau$.

When $A_{\max }$ reaches its lowest value at $\tau=50 \%$, the energy dissipated by dry friction $E_{\text {friction }}$ is the lowest while the out-of-phase force $F_{o / x}$ reaches a maximum.

The effect of the nonlinear force appears clearly when viscous damping is not considered. The maximal amplitude is at resonance and, in this case, the total in-phase force per displacement unit $F_{p / x}$, given by Eq. (22) is classically equal to zero. Without viscous damping, the total out-of-phase force per displacement unit $F_{o / x}$ is only constituted by the cosine component $F_{n l c o s}$ of the nonlinear force:

$$
F_{o / x}=\frac{F_{\text {nlcos }}}{A_{\max }}
$$

Then, the equilibrium given Eq. (5) reduces to:

$$
F_{\text {tot } / x}=\sqrt{F_{o / x}^{2}+F_{p / x}^{2}}=F_{o / x}=\frac{F_{n l c o s}}{A_{\max }}=f_{0} / A_{\max }
$$

Consequently, the maximal amplitude $A_{\max }$ is inversely proportional to the out-of-phase force per displacement.

As a conclusion, changes in contact states during a stick/slip phase has a major influence on the out-of-phase force per displacement unit and consequently on the maximal vibration amplitudes. Then, FRF flattening may not be explained by energy dissipation induced by the rubbing element but by the perturbation in boundary conditions it induces during each vibration cycle. 


\section{Conclusion}

The objective of the present study is directed toward the identification of the physical causes explaining the flattening phenomenon of FRF peaks of structures including dry friction devices. Two different hypotheses, classically found in the literature, have been examined: the first is related to energy dissipated and the second implies changes in contact states during stick/slip motions.

Numerical results, obtained using a representative single degree-of-freedom system, show that peak flattening cannot be associated to an increase in the dissipated energy induced by dry friction nor to an increase in the total dissipated energy (dry friction and viscous damping) as those two quantities decrease with amplitude. Considering dissipation, it should be however noticed that a majority of the whole dissipated energy may be associated to dry friction within stick/slip states.

On the other hand, the analysis of the different forces acting on the system at resonance proves that when contact moves toward stick/slip states, the out-of-phase force per displacement unit increases, implying a decrease in vibration amplitudes. The contact state that minimises amplitudes is half-stuck and half-slip when viscous damping is neglected. When viscous damping is considered and for classical values of damping ratios, the optimal contact state changes slightly towards more stuck configurations. However, also in this case, the increase in the out-of-phase force per displacement unit when contact moves toward stick/slip states is confirmed to be the real cause of peak flattening.

\section{References}

[1] A. Toufine, J.J. Barrau, M. Berthillier, Dynamic study of a structure with flexion-torsion coupling in the presence of dry friction, Nonlinear Dynamics 18 (1999) 321-337.

[2] K. Popp, L. Panning, W. Sextro, Vibration damping by friction forces: theory and applications, Journal of Sound and Vibration 9 (3-4) (2003) 419-448.

[3] B.D. Yang, C.H. Menq, Characterization of 3D contact kinematics and prediction of resonant response of structures having 3D frictional constraint, Journal of Sound and Vibration 217 (5) (1998) 909-925.

[4] R. Masiani, D. Capecchi, F. Vestroni, Resonant and coupled response of hysteretic two-degree-of freedom systems using harmonic balance method, NonLinear Mechanics 37 (2002) 1421-1434.

[5] K.Y. Sanliturk, D.J. Ewins, Modelling two-dimensional friction contact and its application using harmonic balance method, Journal of Sound and Vibration 193 (2) (1996) 211-523.

[6] E.P. Petrov, D.J. Ewins, Analytical formulation of friction interface elements for analysis of nonlinear multiharmonic vibrations of bladed discs, ASME Journal of Turbomachinery 125 (2002) 364-371.

[7] G. Csaba, Forced response analysis in time and frequency domains of a tuned bladed disk with friction dampers, Journal of Sound and Vibration 214 (3) (1998) 395-412.

[8] L. Panning, W. Sextro, K. Popp, Optimization of interblade friction damper designProc. of 2000 ASME Turbo Expo, May 8-11, 2000, Munich, Germany 2000GT-541, 2000.

[9] T. Berruti, S. Filippi, M.M. Gola, S. Salvano, Friction damping of interlocked vane segments: validation of friction model and dynamic responseProc. of 2002 ASME Turbo Expo, June 3-6, 2002, Amsterdam, The Netherlands, 2002-GT-30324, 2002.

[10] E. Chatelet, G. Michon, L. Manin, G. Jacquet, Stick/slip phenomena in dynamics: choice of contact model. Numerical predictions \& experiments, Journal of Mechanism and Machine Theory 43 (2008) 1211-1224.

[11] W. Sextro, The calculation of the forced response of shrouded blades with friction contacts and its experimental verificationProc. of 2000 ASME Turbo Expo, May 8-11, 2000, Munich, Germany 2000-GT-540, 2000.

[12] L. Panning, K. Popp, F. Götting, A. Kayser, I. Wolter, Asymmetrical underplatform dampers in gas turbine blandings: theory and applicationProc. of 2004 ASME Turbo Expo, June 14-17, Vienna, Austria GT 2004-53316, 2004.

[13] T. Berruti, S. Filippi, M.M. Gola, S. Salvano, Friction damping of interlocked vane segments: experimental results, ASME Journal of Engineering for Gas Turbine and Power 124 (2002) 1018-1024.

[14] Mikel Zubieta, Maríajesús Elejabarrieta, M. Mounir, Bou-Ali characterization and modeling of the static and dynamic friction in a damper, Mechanism and Machine Theory 44 (2009) 1560-1569.

[15] Duan, R. Singh, Dynamics of a 3dof torsional system with a dry friction controlled path, Journal of Sound and Vibration 289 (2006) 657-688.

[16] G. Michon, L. Manin, R. Dufour, Hysteretic behaviour of a belt tensioner: modeling and experimental investigation, Journal of Vibration and Control 11 (9) (2005) $1147-1158$.

[17] J.J. Chen, B.D. Yang, C.H. Menq, Periodic forced response of structures having three-dimensional friction constraints, Journal of Sound and Vibration 229 (4) (2000) $775-792$.

[18] Menq C.H. The vibration response of frictionally constrained gas turbine engine blades. Thesis mechanical engineering Pittsburgh, Pennsylvania: CarnegieMellon University, 1985, $172 \mathrm{pp}$.

[19] J.H. Wang, W.K. Chen, Investigation of the vibration of blade with friction damper by HBM, Journal of Engineering for Gas Turbines and Power, Transactions of the ASME 115 (APRIL 1993). 